\title{
Heat treatment of biochemical samples to inactivate Ebola virus: does it work in practice?
}

Hong Kong Med J 2015;21:378-9

DOI: 10.12809/hkmj154629

To the Editor-We read with interest the article by Chong et $\mathrm{al}^{1}$ on the effects of plasma heating procedures on common biochemical tests. Heat treatment at $60^{\circ} \mathrm{C}$ for 60 minutes has also been suggested by our Australian guidelines as a means to inactivate Ebola virus prior to routine laboratory processing. $^{2}$

We recently performed a similar study to Chong et al $^{1}$ in our laboratory at the Royal Melbourne Hospital (Parkville, Australia). De-identified plasma $(n=29)$ and serum $(n=38)$ venous samples were collected in plastic BD vacutainer tubes (Becton, Dickinson and Company, Franklin Lakes [NJ], US) for electrolyte, liver function, and troponin testing on the Architect c16000 analyser (Abbott Laboratories, North Chicago [IL], US). After centrifugation, two $0.5-\mathrm{mL}$ aliquots were obtained, one placed in a heat block at $60^{\circ} \mathrm{C}$ for 60 minutes, and the other paired sample left at room temperature. After heat inactivation, 24/27 (89\%) plasma samples and 25/36 (69\%) serum samples changed to a viscous jelly-like substance (Fig) and caused aspiration error on the Architect analyser. Centrifugation, manual stirring, and vortexing did not resolve the problem.

In serum samples that were not denatured by heating, electrolyte measurements had a strong

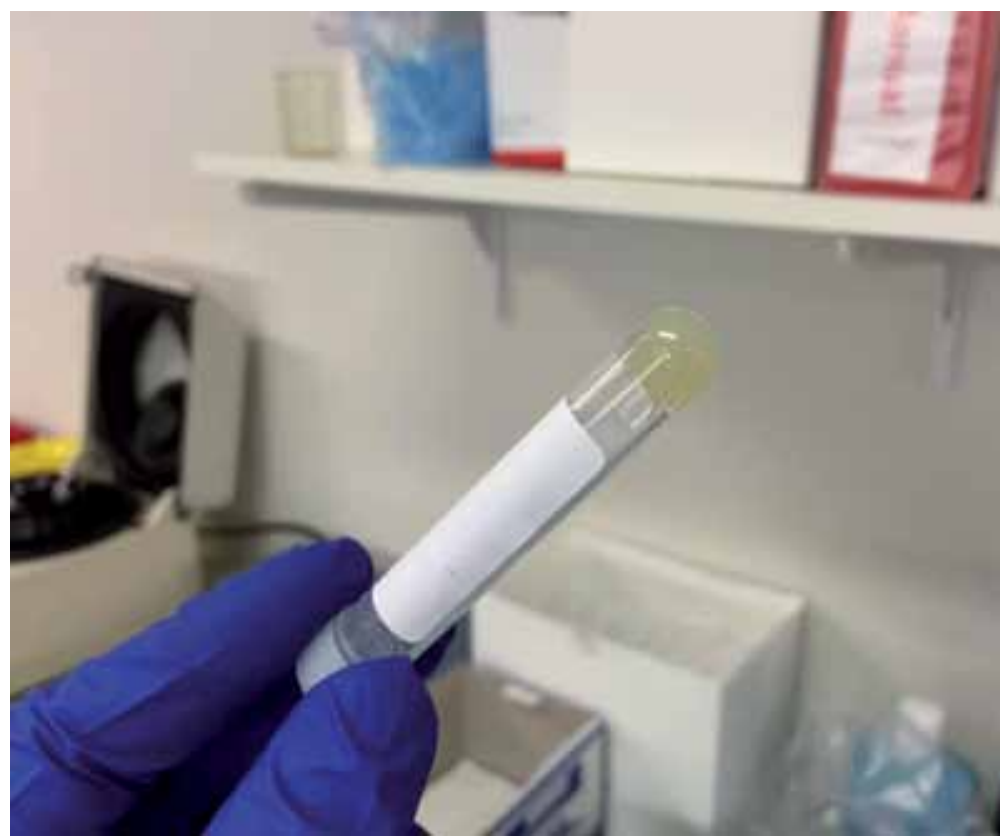

FIG. Heat inactivated plasma: this sample $(0.5 \mathrm{~mL})$ was heat inactivated at $60^{\circ} \mathrm{C}$ for 60 minutes correlation with results obtained by standard testing. Nonetheless, enzyme tests (liver function and troponin) showed a poor correlation (Table). Interestingly, these problems have not been previously reported. ${ }^{2,3}$ The use of a heat block instead of a water bath ${ }^{2}$ may have exposed our specimens to unequal heating, hotspots, or spikes in temperature, causing condensation or irreversible denaturing of proteins. ${ }^{4}$

We found that, in our hands, it was not possible to provide biochemical testing after heat treatment, as recommended by current national and international guidelines.

\section{Timothy B Nguyen, MD}

Vanessa Clifford *, MB, BS

Azni Abdul Wahab, MB, BS

Vincent Sinickas, FRCPA, FRACP

Department of Pathology, Royal Melbourne Hospital, Parkville, Victoria, Australia

* Corresponding author: vanessa.clifford@mh.org.au

\section{References}

1. Chong YK, Ng WY, Chen SP, Mak CM. Effects of a plasma heating procedure for inactivating Ebola virus on common chemical pathology tests. Hong Kong Med J 2015;21:201-7.

2. Public Health Laboratory Network. Laboratory procedures and precautions for samples collected from patients with suspected viral haemorrhagic fevers: Australian Government Department of Health; 2014. Available from: http://www.health.gov.au/internet/main/publishing.nsf/ Content/cda-pubs-other-vhf.htm. Accessed 10 May 2015.

3. Bhagat CI, Lewer M, Prins A, Beilby JP. Effects of heating plasma at 56 degrees $\mathrm{C}$ for $30 \mathrm{~min}$ and at 60 degrees $\mathrm{C}$ for $60 \mathrm{~min}$ on routine biochemistry analytes. Ann Clin Biochem 2000;37:802-4.

4. Wetzel R, Becker M, Behlke J, et al. Temperature behaviour of human serum albumin. Eur J Biochem 1980;104:469-78.

\section{Authors' Reply}

To the Editor-We would like to thank Nguyen et al for their comments.

We concur with Nguyen et al that the use of heat blocks rather than a water bath may result in unequal heating, hotspots, or spikes in temperature. In our previous experience with heating procedures performed to determine alkaline phosphatase isoenzymes, ${ }^{1}$ exposure of plasma to a temperature of $65^{\circ} \mathrm{C}$ caused gelling of most plasma specimens (unpublished observations). We suspect that the gelling temperature of normal human plasma is $60^{\circ} \mathrm{C}$ 
TABLE. Heat inactivation of serum specimens $(n=I I)$ at $60^{\circ} \mathrm{C}$ for 60 minutes compared with matched room temperature controls. All statistical analyses were performed using the Wilcoxon signed rank test, which is the non-parametric method of comparing matched pairs (GraphPad Prism 6.0). A $P$ value of $<0.002$ was considered significant after a Bonferroni adjustment for 21 multiple comparisons

\begin{tabular}{|c|c|c|c|c|c|}
\hline \multirow[t]{2}{*}{ Analyte } & \multicolumn{2}{|c|}{ Room temperature } & \multicolumn{2}{|c|}{ Heat inactivated } & \multirow[t]{2}{*}{$P$ value } \\
\hline & Median & $95 \% \mathrm{Cl}$ & Median & $95 \% \mathrm{Cl}$ & \\
\hline \multicolumn{6}{|l|}{ Electrolytes } \\
\hline Sodium (mmol/L) & 141 & $137.8-143.8$ & 142 & $138.4-145.7$ & 0.168 \\
\hline Potassium (mmol/L) & 4.15 & $3.78-4.48$ & 4.25 & $3.83-4.54$ & 0.07 \\
\hline Chloride (mmol/L) & 104.5 & $101.4-108.7$ & 104 & $102.0-110.0$ & 0.119 \\
\hline Calcium (mmol/L) & 2.25 & $2.00-2.284$ & 2.04 & $1.85-2.16$ & 0.014 \\
\hline Phosphate (mmol/L) & 1.27 & $0.92-1.70$ & 1.12 & $0.82-1.56$ & 0.037 \\
\hline Magnesium (mmol/L) & 0.85 & $0.78-1.01$ & 0.89 & $0.72-0.99$ & 0.016 \\
\hline \multicolumn{6}{|l|}{ Liver function tests } \\
\hline Alkaline phosphatase (U/L) & 80 & $58.73-190.9$ & 5 & $4.54-7.15$ & $<0.001^{\star}$ \\
\hline Alanine aminotransferase (U/L) & 31 & $10.62-122.1$ & 6 & $5.7-6.8$ & $0.001^{*}$ \\
\hline Aspartate aminotransferase (U/L) & 32 & $23.38-62.08$ & 17 & $6.33-32.22$ & $<0.001^{*}$ \\
\hline Gamma-glutamyl transferase (U/L) & 49.5 & $30.90-117.3$ & 4 & $2.90-6.93$ & $<0.001^{*}$ \\
\hline Bilirubin $(\mu \mathrm{mol} / \mathrm{L})$ & 6.28 & $0-70.08$ & 6.73 & $0-70.49$ & 0.032 \\
\hline \multicolumn{6}{|l|}{ Renal function tests } \\
\hline Creatinine $(\mu \mathrm{mol} / \mathrm{L})$ & 94.45 & $80.31-201.1$ & 97.9 & $82.66-204.0$ & 0.065 \\
\hline Urea (mmol/L) & 10.7 & $6.66-16.13$ & 10.75 & $6.76-16.16$ & 0.26 \\
\hline Bicarbonate (mmol/L) & 21.65 & $18.18-25.28$ & 19.25 & $16.82-23.74$ & 0.005 \\
\hline \multicolumn{6}{|l|}{ Cardiac enzymes } \\
\hline Troponin ( $\mu \mathrm{g} / \mathrm{L})$ & 295 & $0-1535$ & 185 & $0-574.7$ & 0.625 \\
\hline Creatine kinase $(\mu \mathrm{mol} / \mathrm{L})$ & 80 & $16.96-300.9$ & 7 & $6.78-7.59$ & $0.001^{*}$ \\
\hline Total protein (g/L) & 61 & $54.61-65.87$ & 63 & $55.61-68.72$ & 0.023 \\
\hline Albumin $(g / L)$ & 34 & $25.67-35.26$ & 25 & $21.47-30.99$ & 0.02 \\
\hline Glucose (mmol/L) & 6.15 & $3.99-10.23$ & 6.15 & $4.24-9.79$ & 0.647 \\
\hline \multicolumn{6}{|l|}{ Indices } \\
\hline Lipaemia & -0.01 & $-0.50-1.30$ & 0.96 & $-0.17-3.55$ & $0.001^{*}$ \\
\hline Icterus & 13.3 & $-2.81-68.36$ & 16.5 & $-19.32-81.05$ & 0.054 \\
\hline Haemolysis & 0.05 & $0.03-1.18$ & 0.08 & $-0.20-0.15$ & 0.008 \\
\hline
\end{tabular}

Abbreviation: $\mathrm{Cl}=$ confidence interval

* $\mathrm{P}<0.002$

to $65^{\circ} \mathrm{C}$.

In our experiments, we use a W14 water bath with 14 L capacity (Sheldon Manufacturing Inc, Cornelius [OR], US). The water bath has a much higher heat capacity than heat blocks, due to the large volume of water, as well as the considerably higher specific heat capacity of water $\left(4.1813 \mathrm{~J} \mathrm{~g}^{-1} \mathrm{~K}^{-1}\right)$ when compared with aluminium $\left(0.897 \mathrm{~J} \mathrm{~g}^{-1} \mathrm{~K}^{-1}\right),{ }^{2}$ a metal often used to manufacture heat blocks.

YK Chong, MB, BS

WY Ng, MB, ChB, PhD

Sammy PL Chen, FRCPA, FHKAM (Pathology)
CM Mak *, FRCPA, FHKAM (Pathology)

Chemical Pathology Laboratory, Department of Pathology, Princess

Margaret Hospital, Laichikok, Hong Kong

* Corresponding author: makm@ha.org.hk

\section{References}

1. Panteghini M, Bais R. Serum enzymes. In: Burtis CA, Ashwood ER, Bruns DE. Tietz textbook of clinical chemistry and molecular diagnostics. St Louis, US: Elsevier; 2012: 579.

2. Chung DD. Composite materials. London: Springer; 2010: 283 